\title{
Chronic dry eye disease is principally mediated by effector memory Th17 cells
}

\author{
Y Chen ${ }^{1}$, SK Chauhan ${ }^{1}$, H Soo Lee ${ }^{1}$, DR Saban ${ }^{1}$ and R Dana ${ }^{1}$
}

Recent experimental and clinical data suggest that there is a link between dry eye disease (DED) and T-cell-mediated immunity. However, whether these immune responses are a consequence or cause of ocular surface inflammation remains to be determined. Thus far, only models of acute DED have been used to derive experimental data. This is in contrast to clinical DED which usually presents as a chronic disease. In the present study, using a murine model of chronic DED, it was established that the chronic phase of the disease is accompanied by Thelper type 17 (Th17) responses at the ocular surface and that a significant memory T-cell population can be recovered from chronic DED. This memory response is predominantly mediated by Th17 cells. Moreover, adoptive transfer of this memory T-cell population was shown to induce more severe and rapidly progressing DED than did the adoptive transfer of its effector or naive counterparts. Not only do these results clearly demonstrate that effector memory Th17 cells are primarily responsible for maintaining the chronic and relapsing course of DED, but they also highlight a potentially novel therapeutic strategy for targeting memory immune responses in patients with DED.

\section{INTRODUCTION}

Dry eye disease (DED) is an extremely common ocular disorder affecting tens of millions of people worldwide ${ }^{1,2}$ and is associated with a diverse array of etiopathogenic factors. ${ }^{3}$ Despite the advent of several therapeutics, notably topical cyclosporine A for DED, there remains a significant unmet medical need. It was only during the past decade that ocular surface inflammation was recognized as a hallmark of DED, ${ }^{3}$ and T-cell infiltration of the ocular surface was identified in a wide spectrum of patients with DED; $;{ }^{4,5}$ however, the immunopathogenic mechanisms that mediate chronic DED have yet to be fully described.

The cellular and molecular mechanisms that underlie inflammation in DED have been investigated with a variety of experimental models. It has been demonstrated that $\mathrm{CD} 4^{+} \mathrm{T}$ cells mediate DED induction in mice; ${ }^{6,7}$ furthermore, $\mathrm{CD} 4{ }^{+}$ T-cell subsets, including T helper type 1 (Th1) and Th17 cells, are now thought to be the major effector cells in DED. ${ }^{8-10}$ Interestingly, regulatory $\mathrm{T}$ cells, which normally suppress immune responses, have also been found to be dysfunctional in DED.$^{10}$ However, it is unclear to what extent these pathogenic findings seen in an acute care setting can be directly related to those found in a clinical setting, where DED is generally encountered as a chronic disorder.

In the present study, we provide, for the first time, a useful animal model of chronic DED and test the hypothesis that the chronic inflammation in DED is mediated by memory $\mathrm{T}$ cells. We examine the immunoinflammatory responses on the ocular surface in chronic DED and characterize the phenotypes of the involved T cells. Furthermore, we determine whether or not the $\mathrm{T}$ cells from chronic DED can adoptively transfer the disease to immunocompetent naive hosts.

\section{RESULTS \\ Chronic DED is characterized by persistent ocular surface inflammation \\ A widely used murine model of $\mathrm{DED}^{11,12}$ was modified to mirror the long-term, fluctuating course of human DED. ${ }^{13}$ Following the initial 14 day of desiccating stress, mice were housed in a standard, non-desiccated vivarium for 4 months without any pharmacological manipulations such as use of scopolamine or other anti-cholinergics (Figure 1a). Clinical disease severity peaked at the end of the desiccating challenges (day 14), as demonstrated by elevated corneal fluorescein}

${ }^{1}$ Department of Ophthalmology, Schepens Eye Research Institute, Massachusetts Eye and Ear Infirmary, Harvard Medical School, Boston, Massachusetts, USA. Correspondence: R Dana (reza.dana@schepens.harvard.edu)

Received 29 October 2012; accepted 7 March 2013; published online 10 April 2013. doi:10.1038/mi.2013.20 

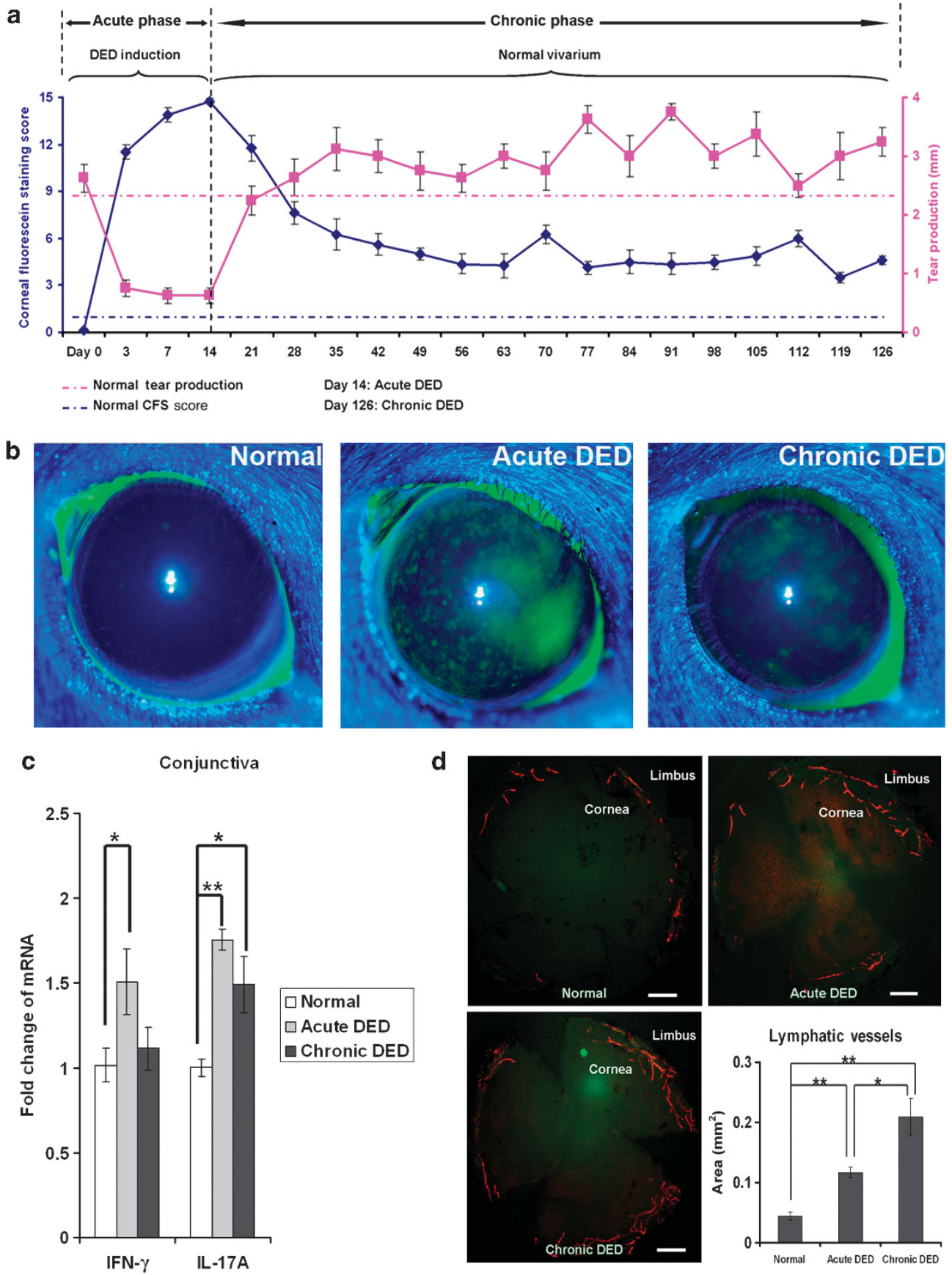

Lymphatic vessels

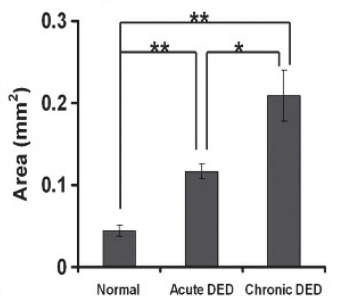

Figure 1 Chronic dry eye disease (DED) involves immunoinflammatory responses at the ocular surface. (a) Development of chronic DED. Acute DED was induced in mice by desiccating stress for 14 days. Subsequently, mice were transferred to the normal vivarium without anti-cholinergic challenge and observed until day 126. Low-level corneal epitheliopathy persisted as evidenced by a slight elevation in corneal fluorescein staining (CFS) scores (solid blue line), which were evaluated in a masked fashion. Meanwhile, aqueous tear production, as assessed with the cotton thread test, returned to normal or even to supra-normal levels (solid pink line). Age- and sex-matched mice maintained in the standard environment were used as normal controls with mean levels of CFS scores and tear production shown in dashed blue and pink lines, respectively. Data shown represent the mean \pm s.e.m. of a single trial ( $n=10$ eyes) out of two performed. (b) Representative CFS images of normal, acute DED (day 14), and chronic DED (day 126). (c) Relative quantification of interferon (IFN)- $\gamma$ and interleukin (IL)-17 mRNA levels in the conjunctiva. Data represent the mean \pm s.e.m. of six eyes per group from a single experiment that was reproduced in a similar independent experiment. ${ }^{\star} P<0.05 ;{ }^{\star \star} P<0.01$. (d) Representative whole-mount corneal immunofluorescence micrographs demonstrating lymphangiogenesis in DED. Corneas were stained with CD31 (green) and LYVE-1 (red) to evaluate blood (CD31 $\left.{ }^{\mathrm{hi}} \mathrm{LYVE}-1^{-}\right)$and lymphatic $\left(\mathrm{CD} 31^{\mathrm{lo}} \mathrm{LYVE}-1^{+}\right)$vessels. Bars $=50 \mu \mathrm{m}$. The corneal area covered by lymphatic vessels were quantified using Fiji software and presented in a bar graph. ${ }^{\star} P<0.05 ;{ }^{\star \star} P<0.01$.

staining scores (a common clinical readout for the severity of corneal epitheliopathy), ${ }^{11,14}$ and decreased aqueous tear secretion. Upon removal from the desiccating environment, corneal epitheliopathy gradually regressed to lower levels, but never normalized, through to the end of the observation period (day 126) (Figure 1a,b). Meanwhile, aqueous tear secretion 
returned to normal, or even to supra-normal levels, indicating that DED with mild corneal epitheliopathy persisted despite the resolution of aqueous tear deficiency or even with an enhanced compensatory tear secretion by the lacrimal apparatus. This demonstrated, for the first time, that following the induction of acute DED, ocular surface inflammation, as characterized by corneal epitheliopathy, persisted into a long-term chronic phase, even without continued exposure to desiccating stress. As corneal epitheliopathy is the most evaluated clinical sign of $\mathrm{DED},{ }^{14,15}$ and absence of tear deficiency is very commonly seen in clinical DED patients, ${ }^{16}$ the persistent ocular surface inflammation indicates the development of a chronic DED state.

\section{Ocular surface inflammation in chronic DED is associated with Th17, but not Th1, immune responses}

We next sought to determine the immunoinflammatory factors that could be responsible for the corneal epitheliopathy observed in chronic DED. Our results in acute DED (day 14) (Figure 1c) were consistent with previous findings, indicating that there is an increased Th1-associated interferon (IFN)- $\gamma^{8}$ and Th17-associated interleukin (IL)-17 in the conjunctiva. ${ }^{8,10}$ However, in chronic DED (day 126), conjunctival IFN- $\gamma$ returned to near-normal levels, while IL-17 levels remained elevated at a level comparable with those found in acute DED (Figure 1c). These findings suggest that chronic DED is associated with a Th17 response. We further examined whether chronic DED is accompanied by corneal angiogenesis. Previous studies from our lab have demonstrated that there is a significant and exclusive growth of lymphatic (not blood) vessels in acute DED corneas ${ }^{17}$ and that the selective lymphangiogenesis in cornea is due to IL-17-driven expression of pro-lymphangiogenic factors. ${ }^{12}$ In the present study, chronic DED corneas did not demonstrate regression but displayed progressive ingrowth of these selectively formed lymphatics toward the center of corneas (Figure 1d).

\section{T cells from chronic DED are pathogenic and can induce the disease}

To determine whether the $\mathrm{T}$ cells generated in DED are indeed pathogenic, we next investigated whether or not the $\mathrm{T}$ cells isolated from chronic DED can induce disease in naive mice. $\mathrm{T}$ cells from the various groups (normal, acute DED, and chronic DED) were adoptively transferred to naive mice, and these mice were subsequently challenged with desiccating environmental stress without scopolamine (Figure 2a). Chronic DED-T-cell recipients developed clinical disease much more rapidly and in a more severe form than did normal (non-DED) T-celltransferred or acute DED-T-cell-transferred recipients (Figure 2b). The highest Th17 responses were observed in recipients of chronic DED-T cells on day 6 after challenge (Figure 2c), suggesting a close link between Th17 response and DED severity. By contrast, there were no increased Th1 responses in chronic DED-T-cell-transferred recipients (Figure 2c). Recipients of acute DED-T cells exhibited only a slightly higher disease score on day 6 after challenge than did recipients of normal T cells (Figure 2b), with comparable Th17 responses (Figure 2c). Although recipients of acute DED-T cells demonstrated the strongest Th1 responses (Figure 2c), this was not paralleled by simultaneous development of clinical disease severity, suggesting that Th17 response was the dominant pathogenic factor.

\section{Chronic DED is characterized by increased effector memory Th17 cells}

Phenotypic and functional assays were employed to further delineate the $\mathrm{CD}^{+}{ }^{+} \mathrm{T}$-cell populations in normal, acute $\mathrm{DED}$, and chronic DED mice. Draining lymph nodes and spleens were harvested, and their $\mathrm{CD} 4{ }^{+}{ }^{\mathrm{T}}$-cell populations were analyzed for naive, effector, and effector memory $\mathrm{T}$ cells, using flow cytometry analysis ${ }^{18}$ (Figure 3a). As expected, normal T cells contained the highest frequencies of $\mathrm{CD} 6 \mathrm{~L}^{+} \mathrm{CD} 44^{\text {lo }}$ naive $\mathrm{T}$ cells $\left(\mathrm{T}_{\mathrm{N}}\right)$, accounting for $50 \%$ of the total $\mathrm{CD} 4^{+}$ population. Compared with normal $\mathrm{T}$ cells, acute DED-T cells contained significantly more $\mathrm{CD} 62 \mathrm{~L}^{-} \mathrm{CD} 44^{\text {lo }}$ effector T cells $\left(\mathrm{T}_{\mathrm{E}}\right)$, while chronic DED-T cells contained the highest frequency of CD62 $\mathrm{L}^{-} \mathrm{CD} 44^{\text {hi }}$ effector memory $\mathrm{T}$ cells $\left(\mathrm{T}_{\mathrm{M}}\right)$. The most prominent fraction in each group (i.e., $\mathrm{T}_{\mathrm{N}}$ from normal, $\mathrm{T}_{\mathrm{E}}$ from acute $\mathrm{DED}$, and $\mathrm{T}_{\mathrm{M}}$ from chronic $\mathrm{DED}$ ) were sorted and functionally characterized. $\mathrm{T}_{\mathrm{N}}$ (naive) exhibited baseline levels of IFN- $\gamma^{+}$and IL- $17^{+}$cell frequencies. Both $\mathrm{T}_{\mathrm{E}}$ (effector) and $\mathrm{T}_{\mathrm{M}}$ (memory) demonstrated increased IFN- $\gamma^{+}$ and $\mathrm{IL}-17^{+}$cell frequencies. Although $\mathrm{T}_{\mathrm{E}}$ and $\mathrm{T}_{\mathrm{M}}$ showed similar frequencies of IFN- $\gamma^{+}$cells, $\mathrm{T}_{\mathrm{M}}$ contained twice as many IL- $17^{+}$cells as $\mathrm{T}_{\mathrm{E}}$. In addition, the frequencies of IL- $17^{+}$ cells in $\mathrm{T}_{\mathrm{M}}$ were several fold higher than those of IFN- $\gamma^{+}$cells (Figure 3b). No differences were noted in $\mathrm{CD} 4{ }^{+} \mathrm{CD} 25^{+} \mathrm{Foxp}^{+}$regulatory $\mathrm{T}$-cell frequencies among the $\mathrm{T}_{\mathrm{N}}, \mathrm{T}_{\mathrm{E}}$, and $\mathrm{T}_{\mathrm{M}}$ groups (data not shown).

\section{Adoptive transfer of effector memory T cells induces severe dry eye and dominant Th17 responses}

Next, to determine whether the $\mathrm{CD} 4{ }^{+} \mathrm{CD}_{22} \mathrm{~L}^{-} \mathrm{CD} 44^{\text {hi }} \mathrm{T}_{\mathrm{M}}$ fraction of chronic DED-T cells can mediate chronic DED, these $\mathrm{T}_{\mathrm{M}}$ were adoptively transferred to naive mice, with $\mathrm{T}_{\mathrm{N}}$ and $\mathrm{T}_{\mathrm{E}}$ as controls, and recipient mice were subjected to desiccating stress (without use of scopolamine; Figure 4a). $\mathrm{T}_{\mathrm{M}}$ recipients exhibited faster onset and significantly higher level of severity of the disease than did recipients of either $\mathrm{T}_{\mathrm{N}}$ or $\mathrm{T}_{\mathrm{E}}$ (Figure $\left.4 \mathrm{~b}\right) . \mathrm{T}_{\mathrm{E}}$ and $\mathrm{T}_{\mathrm{N}}$ recipients experienced similar courses of disease progression except for the timing of disease onset (day 2), when the $T_{E}$ recipients had a higher disease score. The fact that $T_{M}$ contained more IL-17-producing cells and comparable regulatory $\mathrm{T}$ cells at the time of transfer suggests that the higher clinical disease score observed in the recipients of $T_{M}$ was due primarily to the effector memory Th17-driven immune responses. To confirm this hypothesis, ocular surface and draining lymphoid tissues were harvested for examination of Th17/Th1 responses at day 12 after challenge. Both cornea (Figure 4c) and conjunctiva (Figure 4d) from the $\mathrm{T}_{\mathrm{M}}$ recipients demonstrated significantly increased IL-17 but not IFN- $\gamma$ protein levels in comparison to those of the $\mathrm{T}_{\mathrm{N}}$ or $\mathrm{T}_{\mathrm{E}}$ recipients, or naive mice, though the substantial increase of corneal IL-17 expression in the $\mathrm{T}_{\mathrm{M}}$ recipients compared with $\mathrm{T}_{\mathrm{E}}$ recipients did 


\section{a}

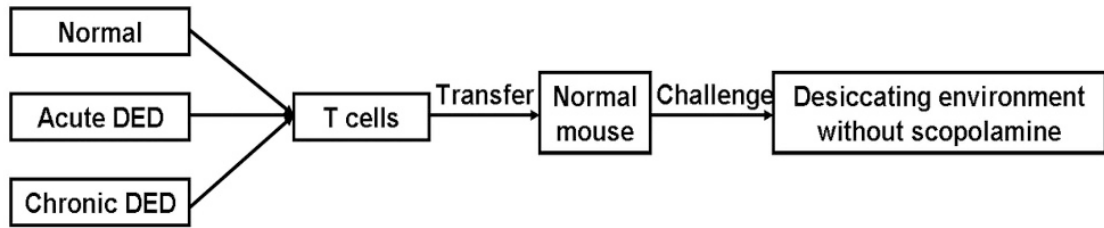

b

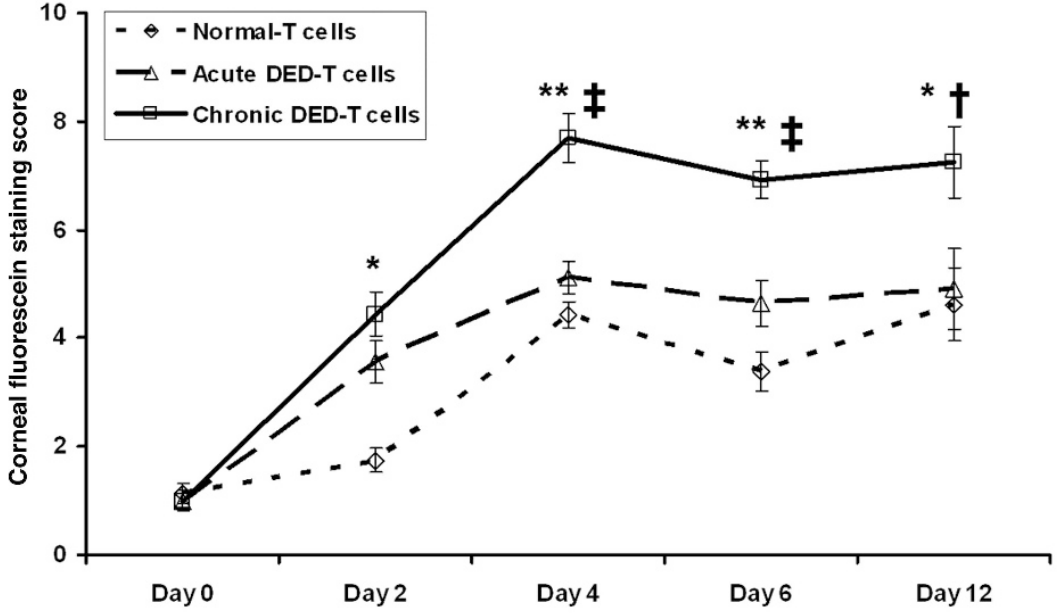

c

\section{Draining LN}

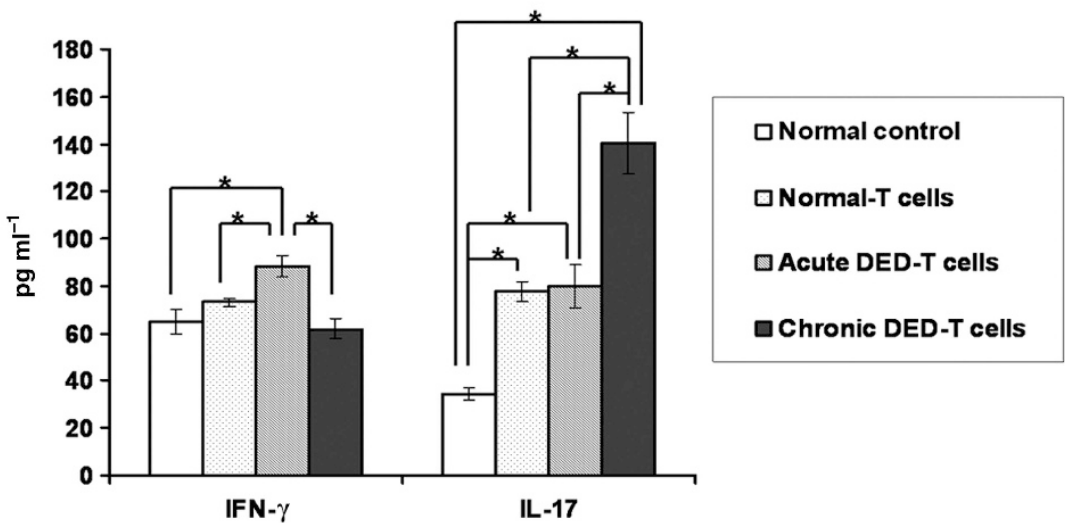

Figure 2 T cells from chronic dry eye disease (DED) mice induce the most severe disease in naive recipients. (a) Schematic study design of adoptive transfer experiment. Total T cells were isolated from normal, acute, and chronic DED ( $n=3-5$ mice/group). Each naive recipient was then injected intravenously with $1 \times 10^{6}$ cells. Recipients were challenged in the controlled-environment chamber for 12 days without use of scopolamine. (b) Disease severity comparison among the recipients transferred with the different $T$ cells. The mean corneal fluorescein staining score \pm s.e.m. for each group ( $n=16$ eyes) is shown. ${ }^{*} P<0.05 ;{ }^{*} P<0.01$ compared with normal-T-cell recipients. ${ }^{\dagger} P<0.05 ;{ }^{\ddagger} P<0.01$ compared with acute DED-T-cell recipients. (c) T-cell response in the recipient draining lymph nodes (LNs). Draining LNs were harvested at day 6 and analyzed for IFN- $\gamma$ and IL-17 levels by enzyme-linked immunosorbent assay ( $n=3$ mice per group). Data shown are mean \pm s.e.m. ${ }^{\star} P<0.05$. Groups are labeled as follows: Normal T-cells, recipients of T cells from normal mice; Acute DED-T cells, recipients of T cells from acute DED mice; and Chronic DED-T cells, recipients of $T$ cells from chronic DED mice.

not reach statistical significance. In concordance with disease severity, $\mathrm{T}_{\mathrm{N}}$ and $\mathrm{T}_{\mathrm{E}}$ recipients showed similar increases in IFN- $\gamma$ and IL-17. Consistent with the ocular surface findings, the draining lymph nodes of $\mathrm{T}_{\mathrm{M}}$ recipients exhibited the highest frequencies of Th17 cells (Figure 4e).

\section{DISCUSSION}

Herein, we have demonstrated that chronic DED involves persistent ocular surface inflammation associated with a Th17 response. A significant effector memory Th17 population can be recovered from chronic DED and shown to mediate the long-term course of the disease.

The current study reveals, for the first time, that the corneal epithelial damage mediated by ocular surface inflammation in DED persists long term. However, the underlying immune response changes with time. Although the acute phase of DED exhibits both Th1 and Th17 responses as indicated in previous reports $^{8,10}$ and the present study, the chronic phase of DED only is mediated by a Th17 response alone, including increased IL-17 expression and lymphangiogenesis on the ocular surface. 


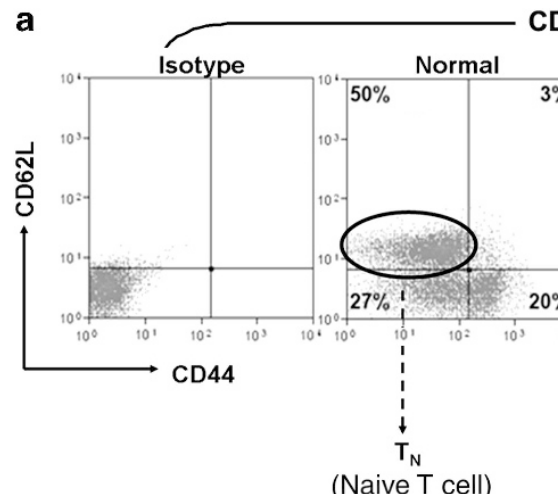

CD4+ gated

(Naive T cell)
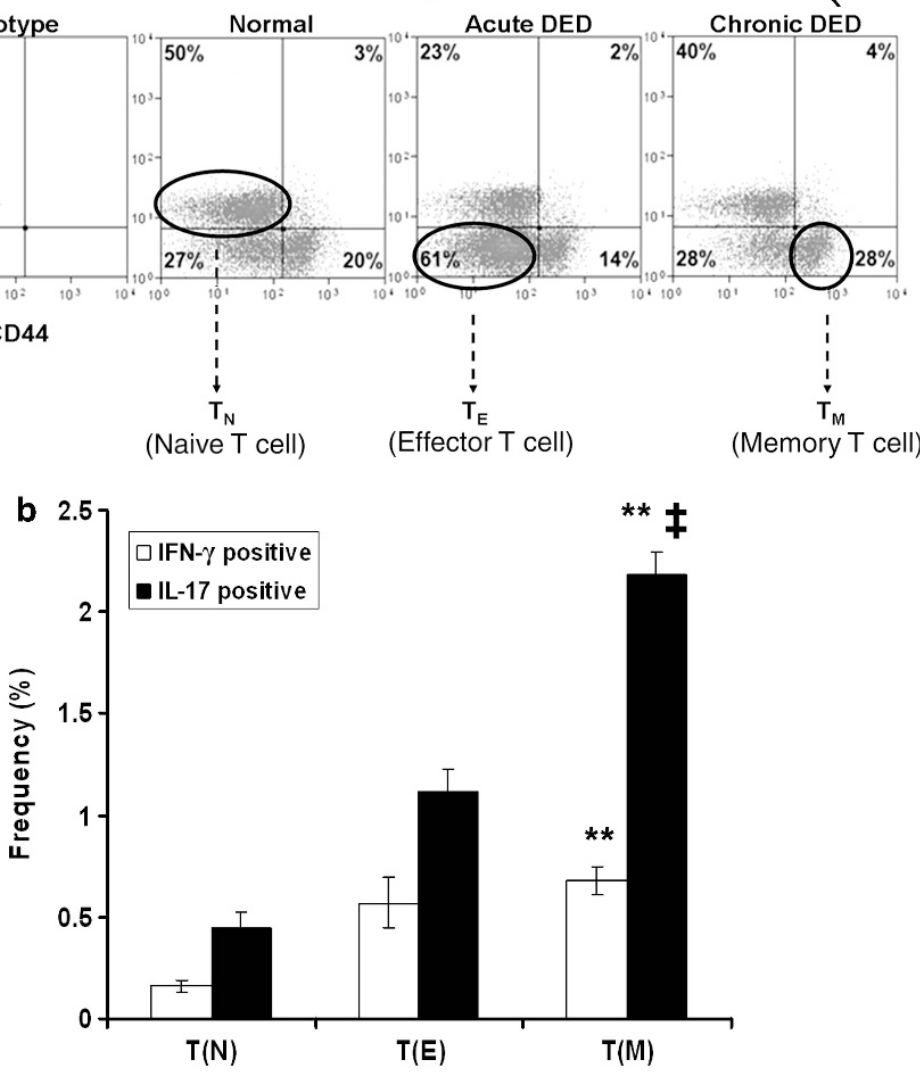

Figure 3 T cells from chronic dry eye disease (DED) show increased effector memory Thelper type 17 population. Draining lymph node cells and spleen cells from normal, acute, and chronic DED mice were analyzed by flow cytometry. Representative results from spleens are shown, with similar findings in draining lymph nodes. (a) CD62L vs. CD44 expression on gated CD4 ${ }^{+}$cells is presented. Four T-cell populations were differentiated: ${ }^{16}$

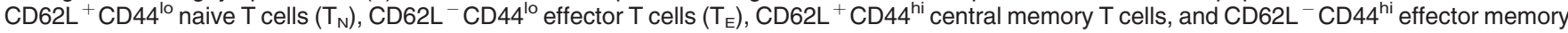
$T$ cells $\left(T_{M}\right)$. Percentages indicate frequencies of each cell population. (b) Sorted $T_{N}$ from normal, $T_{E}$ from acute, and $T_{M}$ from chronic $D E D$ were further analyzed for interferon (IFN)- $\gamma$ and interleukin (IL)-17 expression. Frequencies of IFN- $\gamma^{+}$or IL-17 ${ }^{+}$cells in each individual populations are summarized as mean \pm s.e.m. of three independent experiments shown in the bar graph. ${ }^{\star \star} P<0.01$ compared with $\mathrm{T}_{\mathrm{N}}$; ${ }^{\ddagger} P<0.01$ compared with $\mathrm{T}_{\mathrm{E}}$.

These lymphatic vessels are potential conduits for immune cell migration between the ocular surface and draining lymphoid tissues. ${ }^{19}$ We have noted that the selective lymphangiogenesis in cornea is due to IL-17-driven expression of pro-lymphangiogenic factors. ${ }^{12}$ Therefore, we speculate that the persistent and progressively ingrowing lymphatic vessels in chronic DED corneas are due, at least in part, to the elevated levels of IL-17. Together, these findings suggest that relentless Th17 response at the ocular surface, even in the absence of triggering factors, could be one of the critical inflammatory pathways operating in the vicious cycle of DED. ${ }^{3}$

Previously, Yoon et al. ${ }^{20}$ found that some parameters of experimental DED severity normalize following the removal of desiccating stress. In their study, acute DED was induced using a methodology similar to ours, for 10 days, followed by maintenance in the standard vivarium for 28 days. They found that tear production, corneal smoothness, conjunctival goblet cell density, and conjunctival CD $4{ }^{+}$T-cell density normalized by day 28 in the standard vivarium. However, that study had several limitations. Clinically, the authors did not perform the important corneal epithelial defect assessment using corneal fluorescein staining, as is recommended by the National Eye
Institute/Industry workshop for DED evaluation. ${ }^{14}$ Furthermore, although the numbers of conjunctival CD $4{ }^{+}$T cells were counted, their cytokine secretion function was not examined.

In addition to the experimental data provided herein, several other clinical reports have indicated that DED is associated with a T-cell-mediated immune response, as evidenced by increased infiltration of T cells in the conjunctiva ${ }^{4}$ and elevated levels of T-cell cytokines, including IFN- $\gamma^{21}$ and IL- $17^{22}$ in the tears. However, it is not known whether this immune response is a cause or consequence of DED. In the present study, we demonstrate that $\mathrm{T}$ cells from the chronic DED mice can efficiently induce the disease in the naive host, indicating that these $\mathrm{T}$ cells are pathogenic during the course of the disease. Further phenotypic analysis of the $\mathrm{T}$ cells shows a significant effector memory population, where secretion of IL-17 predominates over that of IFN- $\gamma$. This finding is consistent with the most recent recognition on the nature of Th17 cells as longlived effector memory cells. ${ }^{23,24}$ Previously, it was assumed that both human and mouse Th17 cells are short-lived effector $\mathrm{T}$ cells. However, a recent study on human Th17 cells reveals that these cells have a high capacity for proliferative self-renewal, potent persistence, and apoptotic resistance. ${ }^{23}$ Subsequently, 
a

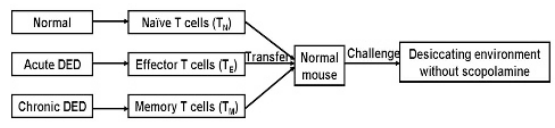

C

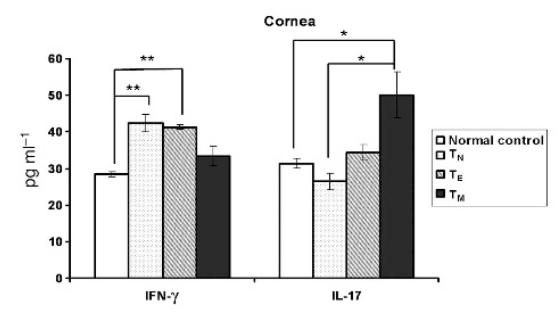

b
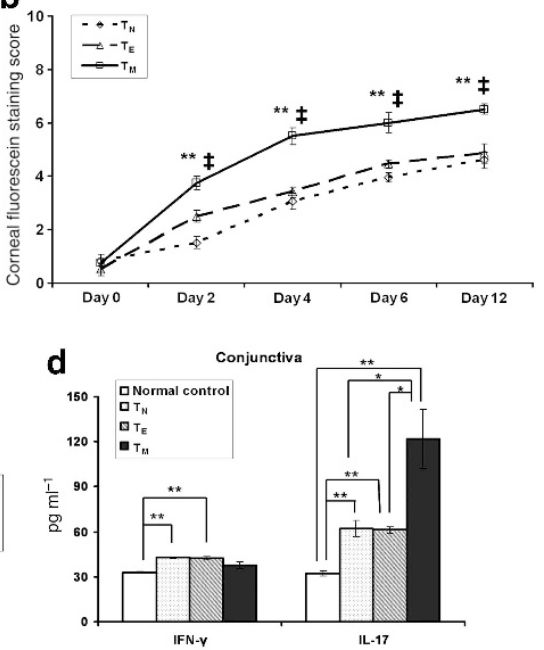
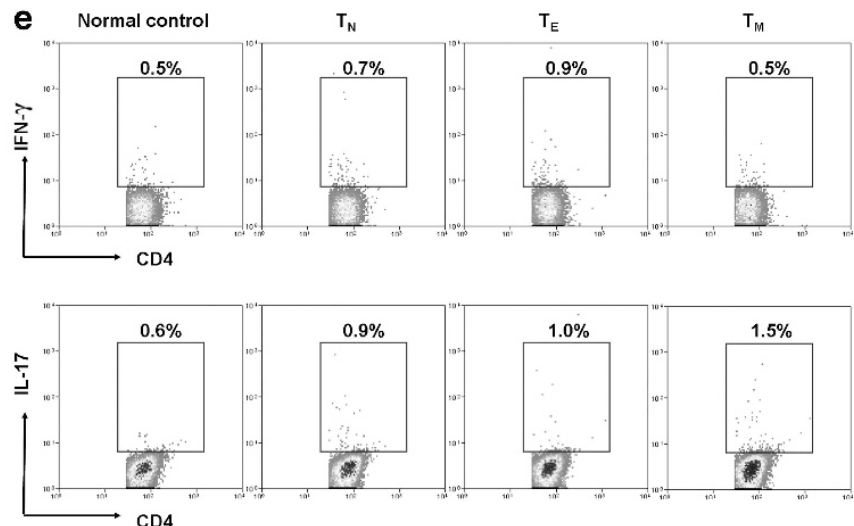

Figure 4 Effector memory T cells $\left(T_{M}\right)$ from chronic dry eye disease (DED) mice induce the most severe disease in naive recipients. (a) Schematic study design of adoptive transfer experiment. The draining lymph nodes and spleens from normal, acute, and chronic DED mice were harvested.

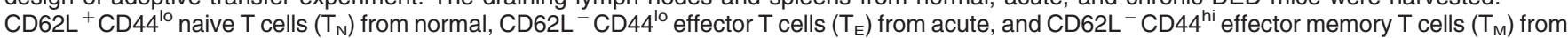
chronic DED were sorted with CD4 beads (negative selection) and fluorescence-activated cell sorter. Each naive recipient was then injected intravenously with $3 \times 10^{5} \mathrm{~T}_{\mathrm{N}}, \mathrm{T}_{\mathrm{E}}$, or $\mathrm{T}_{\mathrm{M}}$ cells. Recipients were challenged with the controlled-environment chamber for 12 days without use of scopolamine. (b) Clinical disease evaluation among the recipients transferred with different cells. The mean corneal fluorescein staining scores \pm s.e.m. for $\mathrm{T}_{\mathrm{N}}\left(n=10\right.$ eyes), $\mathrm{T}_{\mathrm{E}}\left(n=12\right.$ eyes), or $\mathrm{T}_{\mathrm{M}}\left(n=8\right.$ eyes) recipients are shown. ${ }^{*} P<0.05 ;{ }^{* *} P<0.01$ compared with $\mathrm{T}_{\mathrm{N}}$ recipients; ${ }^{\ddagger} P<0.01$ compared with $\mathrm{T}_{E}$ recipients. (c, d) T-cell response at the recipient ocular surface. Cornea (c) and conjunctiva (d) were collected from the recipient mice in $\mathbf{b}$ at day 12 and analyzed for interferon (IFN)- $\gamma$ and interleukin (IL)-17 levels by enzyme-linked immunosorbent assay. Data shown represent the mean $\pm s$.e.m. of a single experiment out of two performed ( $n=5-9$ eyes per group). ${ }^{*} P<0.05 ;{ }^{* \star} P<0.01$. (e) T-cell response in the recipient draining lymph nodes. Single-cell suspension was prepared from lymph nodes and analyzed by flow cytometry for IFN- $\gamma^{+} \mathrm{CD} 4^{+}$Th 1 and IL- $17^{+} \mathrm{CD} 4^{+}$Th 17 cells. The indicated percentages of T helper type 1 (Th1) or Th17 cells as a proportion of total CD4 ${ }^{+} \mathrm{T}$ cells in the representative flow cytometry dot plots were measured in different recipient groups. Groups are labeled as follows: $T(N)$, recipients of naive T cells from normal mice; $T(E)$, recipients of effector T cells from acute DED mice; and $T(M)$, recipients of effector memory $T$ cells from chronic DED mice.

mouse Th17 cells have been found not only to be long lived but also to be empowered with an enhanced ability to enter the memory pool. ${ }^{24}$ These novel discoveries support the association of Th17 cells with various autoimmune diseases such as multiple sclerosis, Crohn's disease, uveitis, and graft-versus-host disease, ${ }^{25-28}$ and support their proposed long-term anti-tumor activity. ${ }^{29,30}$

Next, we examined whether or not the effector memory T-cell population contributes to the protracted damage to the ocular surface identified in chronic DED. Compared with the transfer of effector $\mathrm{T}$ cells to naive recipients, effector memory $\mathrm{T}$ cells elicited a more severe DED, which was similar to total $\mathrm{T}$-cell transfer, indicating that effector memory $\mathrm{T}$ cells is the principal subpopulation mediating the disease. However, effector T cells also induced a higher disease score than did naive $\mathrm{T}$ cells only at disease onset, suggesting that effector $\mathrm{T}$ cells act for a short time, possibly because of their susceptibility to activation-induced cell death. ${ }^{31}$ By contrast, effector memory $\mathrm{T}$ cells demonstrated a strong and lasting effect on the disease, which can be attributed to their rapid expansion and resistance to apoptosis. ${ }^{32,33}$

In summary, the current work demonstrates that the persistent immunoinflammatory responses in chronic DED exhibit hallmarks of IL-17-mediated pathology and implicates effector memory Th17 cells as the principal effector cells in maintaining chronic DED. These findings suggest a pathogenic role of autoimmunity in DED and expand our understandings 
of memory T-cell response in chronic disease states. Accordingly, immunomodulatory therapies that target effector memory Th17 cells may effectively treat DED.

\section{METHODS}

Animals. Female C57BL/6 mice aged 6-8 weeks (Charles River Laboratories, Wilmington, MA) were used for this study. All animal experiments were approved by the Schepens Eye Research Institute Animal Care and Use Committee and adhered to the Association for Research in Vision and Ophthalmology Statement for the Use of Animals in Ophthalmic and Vision Research.

DED models. Acute DED was induced in mice as previously described, with some modification. ${ }^{11}$ Mice were placed in a controlled-environment chamber with a relative humidity $<20 \%$, airflow of $151 \mathrm{~min}^{-1}$, and a constant temperature of $21-23^{\circ} \mathrm{C}$, for 14 consecutive days. To maximize ocular surface dryness, mice received subcutneous $0.1-\mathrm{ml}$ injections of $5 \mathrm{mg} \mathrm{ml}^{-1}$ scopolamine hydrobromide (Sigma-Aldrich, St. Louis, MO). Chronic DED was developed by transferring acute DED mice to a standard vivarium (temperature: $21-23{ }^{\circ} \mathrm{C}$, relative humidity: $\left.40-60 \%\right)$ and maintained for an additional 4 months without any environmental or anti-cholinergic (scopolamine) desiccating challenge.

Clinical evaluation. Corneal fluorescein staining and the National Eye Institute grading system (Bethesda, MD) was used to evaluate corneal epithelial damage caused by DED. ${ }^{14}$ Briefly, $1 \mu \mathrm{l}$ of $2.5 \%$ fluorescein (Sigma-Aldrich) was applied into the lateral conjunctival sac of the mice and after 3 min corneas were examined with a slit lamp biomicroscope under cobalt blue light. Punctate staining was recorded in a masked fashion with the standard National Eye Institute grading system of $0-3$ for each of the five areas of the corneacentral, superior, inferior, nasal, and temporal. The cotton thread test was used to evaluate aqueous tear production as previously described. ${ }^{11}$ Briefly, a phenol red thread (Zone-Quick; Lacrimedics, Eastsound, WA) was placed in the lateral canthus of the conjunctival fornix of the right eye for $30 \mathrm{~s}$ after excess tears had been removed for a standard time of $4 \mathrm{~s}$, and tear distance (in millimeters) was read under a microscope.

Immunohistochemical staining. Freshly excised corneas were washed in phosphate-buffered saline, fixed in acetone for $15 \mathrm{~min}$, and double stained with CD31 and LYVE-1 antibodies (Santa Cruz Biotechnology, Santa Cruz, CA). Subsequently, the corneas were mounted using VECTASHIELD mounting medium (Vector Laboratories, Burlingame, CA) and visualized under an epifluorescence microscope (Model E800; Nikon, Melville, NY) with a $\times 2$ objective lens at $\times 20$ magnification, and then the digitial micrographs were captured. Contrast of these acquired micrographs were linearly adjusted using Photoshop CS2 (Adobe, San Jose, CA) before the lymphatic area was calculated using Fiji software (http://fiji.sc/Fiji), which is an open source image-processing package based on ImageJ software (National Institutes of Health, Bethesda, MD). ${ }^{34,35}$

Real-time PCR. Corneas and conjunctivae from mice were harvested, frozen in TRIzol Reagent (Invitrogen, Carlsbad, CA) and stored at $-80^{\circ} \mathrm{C}$ until use. Total RNA was isolated with an RNeasy Micro kit (Qiagen, Valencia, CA) according to the manufacturer's recommendations and reverse transcribed using a SuperScript III kit (Invitrogen). Real-time PCR was performed using TaqMan Universal PCR Master Mix (Roche, Branchburg, NJ) and predesigned primers for IL-1 $\beta$ (Mm00434228_m1), IL-23 (Mm00518984_m1), IFN- $\gamma$ (Mm00801778_m1), IL-17 (Mm00439619_m1), and glyceraldehyde 3-phosphate dehydrogenase (GAPDH; Mm99999915_g1; Applied Biosystems, Foster City, CA) in a LightCycler 480 II System (Roche Applied Science, Indianapolis, IN). The GAPDH gene was used as an endogenous control for each reaction. The results of quantitative PCR were analyzed by the comparative $\mathrm{C}_{\mathrm{T}}$ method in which the target change was equal to $2^{-\Delta \Delta C T}$. The results were normalized by the $C_{T}$ value of GAPDH, and the mean $\mathrm{C}_{\mathrm{T}}$ of relative mRNA levels in the normal group was used as the calibrator.

Enzyme-linked immunosorbent assay. For protein extraction, corneas, conjunctivae, and draining lymph nodes were harvested and stored in cold sterile phosphate-buffered saline containing protease inhibitors (Sigma-Aldrich) at $-80^{\circ} \mathrm{C}$ until use. The samples were homogenized on ice and centrifuged. The supernatant was assayed for the levels of IFN- $\gamma$ and IL-17 using commercial ELISA kits (eBioscience, San Diego, CA).

Flow cytometry analysis. Cells were triple stained with the following antibodies: fluorescein isothiocyanate (FITC)-conjugated anti-CD4, allophycocyanin-conjugated anti-CD44, and phycoerythrin (PE)-conjugated anti-CD62L (BioLegend, San Diego, CA). For intracellular IFN- $\gamma$ and IL-17 staining, cells were stimulated with $50 \mathrm{ng} \mathrm{ml}^{-1}$ phorbol 12myristate 13 -acetate and $500 \mathrm{ng} \mathrm{ml}^{-1}$ ionomycin (Sigma-Aldrich) for $6 \mathrm{~h}$ at $37^{\circ} \mathrm{C}$ and $5 \% \mathrm{CO}_{2}$ in the presence of GolgiStop ( $4 \mu \mathrm{l}$ per $6 \mathrm{ml}$ cell culture; BD Biosciences, San Jose, CA) to inhibit cytokine secretion. The cells were then stained for FITC-conjugated anti-CD4, allophycocyaninconjugated anti-INF- $\gamma$, and PE-conjugated anti-IL-17 or PE-Cy5-conjugated anti-CD4, FITC-conjugated anti-CD44, PE-conjugated antiCD62L, Alexa Fluor 700-conjugated anti-INF- $\gamma$, and PE-Cy7-conjugated anti-IL-17 (BioLegend). Control samples were stained with appropriate isotype-matched control antibodies. Stained cells were examined with an LSR II Flow Cytometer (BD Biosciences), and the results were analyzed using Summit v4.3 software (Dako Colorado, Fort Collins, CO).

T-cell adoptive transfer. Draining lymph node and spleen cells from normal, acute DED, and chronic DED mice were pooled separately and enriched for T cells with CD90.2 magnetic cell isolation kit (Miltenyi Biotec, Cambridge, MA). In all, $1 \times 10^{6}$ cells were then adoptively transferred intravenously into each naive recipient. For fractional T-cell adoptive transfer, draining lymph node and spleen cells from normal, acute DED, and chronic DED mice were pooled separately and enriched for $\mathrm{CD} 4{ }^{+} \mathrm{T}$ cells with a negative magnetic cell isolation kit (Miltenyi Biotec). The $\mathrm{CD}^{+}{ }^{+} \mathrm{T}$ cells were then quantified, stained with FITC-conjugated anti-CD44 and PE-conjugated anti-CD62L, and sorted for naive $\left(\mathrm{T}_{\mathrm{N}}\right)$, effector $\left(\mathrm{T}_{\mathrm{E}}\right)$, and effector memory $\left(\mathrm{T}_{\mathrm{M}}\right) \mathrm{T}$ cells using a MoFlo Fluorescence Activated Cell Sorter (Dako Cytomation, Carpinteria, CA). In all, $5 \times 10^{5}$ cells were adoptively transferred intravenously into each naive recipient.

Statistical analyses. An unpaired, two-tailed Student's $t$ test was used, and differences were considered significant at $P<0.05$.

\section{ACKNOWLEDGEMENTS}

We thank Dr William Stevenson and Gale Unger, Schepens Eye Research Institute, Boston, MA for critical reading of this manuscript, and Dr SangMok Lee, Schepens Eye Research Institute, Boston, MA for quantitative analysis of corneal lymphatic vessels. This work was supported by NIH grant EY20889.

\section{DISCLOSURE}

The authors declared no conflict of interest.

c 2014 Society for Mucosal Immunology

\section{REFERENCES}

1. Schaumberg, D.A., Sullivan, D.A., Buring, J.E. \& Dana, M.R. Prevalence of dry eye syndrome among US women. Am. J. Ophthalmol. 136, 318-326 (2003).

2. Schaumberg, D.A., Dana, R., Buring, J.E. \& Sullivan, D.A. Prevalence of dry eye disease among US men: estimates from the Physicians' Health Studies. Arch. Ophthalmol. 127, 763-768 (2009). 
3. Subcommittee of the international Dry Eye Workshop. The definition and classification of dry eye disease: report of the definition and classification. Ocul. Surf. 5, 75-92 (2007).

4. Stern, M.E. et al. Conjunctival T cell subpopulations in Sjögren's and non-Sjögren's patients with dry eye. Invest. Ophthalmol. Vis. Sci. 43, 2609-2614 (2002)

5. Kunert, K.S., Tisdale, A.S., Stern, M.E., Smith, J.A. \& Gipson, I.K. Analysis of topical cyclosporine treatment of patients with dry eye syndrome: effect on conjunctival lymphocytes. Arch. Ophthalmol. 118, 1489-1496 (2000).

6. Niederkorn, J.Y. et al. Desiccating stress induces Tcell-mediated Sjögren's Syndrome-like lacrimal keratoconjunctivitis. J. Immunol. 176, 3950-3957 (2006).

7. Schaumburg, C.S. et al. Ocular surface APCs are necessary for autoreactive $T$ cell-mediated experimental autoimmune lacrimal keratoconjunctivitis. J. Immunol. 187, 3653-3662 (2011).

8. De Paiva, C.S. et al. IL-17 disrupts corneal barrier following desiccating stress. Mucosal Immunol. 2, 243-253 (2009).

9. El Annan, J., Chauhan, S.K., Ecoiffier, T., Zhang, Q., Saban, D.R. \& Dana, R. Characterization of effector $\mathrm{T}$ cells in dry eye disease. Invest. Ophthalmol. Vis. Sci. 50, 3802-3807 (2009).

10. Chauhan, S.K. et al. Autoimmunity in dry eye is due to resistance of Th17 to Treg suppression. J. Immunol. 182, 1247-1252 (2009).

11. Barabino, S., Shen, L., Chen, L., Rashid, S., Rolando, M. \& Dana, M.R. The controlled-environment chamber: a new mouse model of dry eye. Invest. Ophthalmol. Vis. Sci. 46, 2766-2771 (2005).

12. Chauhan, S.K. et al. A novel pro-lymphangiogenic function for Th17/IL-17. Blood 118, 4630-4634 (2011).

13. Friedman, N.J. Impact of dry eye disease and treatment on quality of life. Curr. Opin. Ophthalmol. 21, 310-316 (2010).

14. Lemp, M.A. Report of the National Eye Institute/Industry workshop on clinical trials in dry eyes. CLAO J. 21, 221-232 (1995).

15. Mokhtarzadeh, M., Casey, R. \& Glasgow, B.J. Fluorescein punctate staining traced to superficial corneal epithelial cells by impression cytology and confocal microscopy. Invest. Ophthalmol. Vis. Sci. 52, 2127-2135 (2011).

16. Sullivan, B.D. et al. Correlations between commonly used objective signs and symptoms for the diagnosis of dry eye disease: clinical implications. Acta Ophthalmol., advance online publication, 28 December 2012; doi:10.1111/aos.12012 (2012).

17. Goyal, S., Chauhan, S.K., El Annan, J., Nallasamy, N., Zhang, Q. \& Dana, $R$. Evidence of corneal lymphangiogenesis in dry eye disease: a potential link to adaptive immunity?. Arch. Ophthalmol. 128, 819-824 (2010).

18. Seddon, B., Tomlinson, P. \& Zamoyska, R. Interleukin 7 and Tcell receptor signals regulate homeostasis of CD4 memory cells. Nat. Immunol. 4, 680-686 (2003).
19. Goyal, S., Chauhan, S.K. \& Dana, R. Blockade of prolymphangiogenic vascular endothelial growth factor $\mathrm{C}$ in dry eye disease. Arch. Ophthalmol. 130, 84-89 (2012).

20. Yoon, K.C. et al. Tear production and ocular surface changes in experimental dry eye after elimination of desiccating stress. Invest. Ophthalmol. Vis. Sci. 52, 7267-7273 (2011).

21. Massingale, M.L., Li, X., Vallabhajosyula, M., Chen, D., Wei, Y. \& Asbell, P.A. Analysis of inflammatory cytokines in the tears of dry eye patients. Cornea 28, 1023-1027 (2009).

22. Kang, M.H., Kim, M.K., Lee, H.J., Lee, H.I., Wee, W.R. \& Lee, J.H. Interleukin-17 in various ocular surface inflammatory diseases. J. Korean Med. Sci. 26, 938-944 (2011).

23. Kryczek, l. et al. Human TH17 cells are long-lived effector memory cells. Sci. Transl. Med. 12, 104ra100 (2011).

24. Muranski, P. et al. Th17 cells are long lived and retain a stem cell-like molecular signature. Immunity 35, 972-985 (2011).

25. Tzartos, J.S. et al. Interleukin-17 production in central nervous systeminfiltrating Tcells and glial cells is associated with active disease in multiple sclerosis. Am. J. Pathol. 172, 146-155 (2008).

26. Maynard, C.L. \& Weaver, C.T. Intestinal effector T cells in health and disease. Immunity 31, 389-400 (2009).

27. Amadi-Obi, A. et al. TH17 cells contribute to uveitis and scleritis and are expanded by IL-2 and inhibited by IL-27/STAT1. Nat. Med. 13, 711-718 (2007).

28. Kappel, L.W. et al. IL-17 contributes to CD4-mediated graft-versus-host disease. Blood 22, 945-952 (2009).

29. Martin-Orozco, N. et al. Thelper 17 cells promote cytotoxic Tcell activation in tumor immunity. Immunity 31, 787-798 (2009).

30. Gattinoni, L., Powell, D.J. Jr., Rosenberg, S.A. \& Restifo, N.P. Adoptive immunotherapy for cancer: Building on success. Nat. Rev. Immunol. 6, 383-393 (2006).

31. Elyaman, W. et al. Distinct functions of autoreactive memory and effector $\mathrm{CD} 4+\mathrm{T}$ cells in experimental autoimmune encephalomyelitis. Am. J. Pathol. 173, 411-422 (2008).

32. Taylor, J.J. \& Jenkins, M.K. CD4 + memory T cell survival. Curr. Opin. Immunol. 23, 319-323 (2011).

33. van Leeuwen, E.M., Sprent, J. \& Surh, C.D. Generation and maintenance of memory CD4(+) T Cells. Curr. Opin. Immunol. 21, 167-172 (2009).

34. Schindelin, J. et al. Fiji: an open-source platform for biological-image analysis. Nat. Methods 9, 676-682 (2012).

35. Chung, E.S. et al. Contribution of macrophages to angiogenesis induced by vascular endothelial growth factor receptor-3-specific ligands. Am. J. Pathol. 175, 1984-1992 (2009). 